\title{
Modeling and simulation of a cost-effective microfluidic circuit for particles' manipulation
}

\author{
Vito Di Virgilio, Arnau Coll, Sandra Bermejo, Luis Castañer (SM '92) \\ Universitat Politècnica de Catalunya, \\ MNT - Micro and Nano Technology Group \\ C/Jordi Girona 1-3 08034 Barcelona, Spain \\ e-mail:vito@eel.upc.edu
}

\begin{abstract}
In this paper we propose the modeling and simulation using Comsol 3.5a of a microfluidic chip, capable to run several tasks such as focusing, mixing, sorting and trapping nano and microbeads.The microfluidic chip, based on Polydimethyl siloxane (PDMS) and Polyethylenenaphtalate (PEN), is cost effective, flexible and totally biocompatible. The fluidic part is controlled both by pressure driven flow and electrostatics. The manipulation of the beads is purely electrostatic and it is based on a mixed approach: electrophoretic (EP) and dielectrophoretic (DEP). The simulations are run in Comsol 3.5a using basically the MEMS module. The nano and microbeads used are metallic (gold) and dielectric (polystyrene, fused silica).
\end{abstract}

Keywords-component; microfluidics; electrophoresys; nanobeads manipulation/trapping; PDMS

\section{INTRODUCTION}

In the last two decades microfluidic systems have gained a very important role in many research fields. Integrated microfluidic systems, generally called "micro total analysis systems" ( $\mu \mathrm{TAS}$ ), have demonstrated to be extremely powerful as biological essay platforms.

DNA sequencing, polymerase chain reaction (PCR), electrophoresis, DNA separation, protein analysis, immunoassay, cell counting, cell sorting, and cell culture are some important tasks which have been successfully integrated on microfluidic lab-on-a-chip (LOC) [1-5].

The miniaturization of the analysis systems allow to use smaller quantities of reagents and speed up the time process. In addition the power consumption decreases as the electric potential applied is smaller. Those reasons make microfluidic systems attractive and potentially portable for in-situ analysis [6]. In order to enhance the reactions and facilitate the signal collection and processing, micro and nanobeads have been integrated [6] in LOCs because they are a powerful reagent support as they show a large surface area, can be easily functionalized and show a high chemical and mechanical stability; in addition they can be easily manipulated and placed in desired places within the microfluidic chip.

We report here the design of a microfluidic holder and the modeling and simulation, using Comsol 3.5a, of a microfluidic total system analysis, completely based on flexible cheap polymers, easy to fabricate, biocompatible and cost effective, capable to manipulate micro and nanobeads.

A conventional medical syringe provides the pressure driven flow. The focusing flow is obtained by lateral flow.
The electrodes are directly obtained depositing and micromachining a thin metal layer on PEN.

The micromachining of the electrodes is obtained by metal laser ablation, directly writing the electrodes on a metal thin layer deposited on a glass substrate.

The external electrical signals are provided to the lab-on-achip by a custom inexpensive patch clamp holder, designed and realized using a PMMA block, a commercial drill, a PCB and pogopins.

\section{HOLDER'S SETUP}

The microfluidic chip is interfaced by a custom PMMA patch clamp holder, figure 1 . The liquid is supplied by a

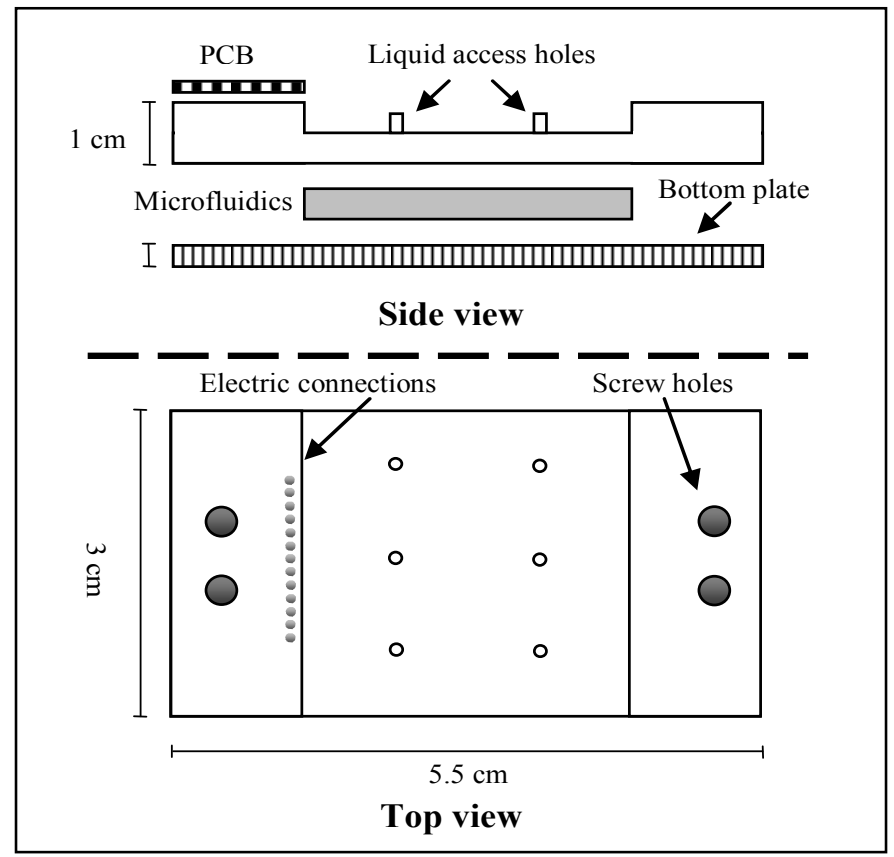

Figure 1. Holder setup scheme: the side view highlights the position of the PCB for electric signals transfer and the position of the microfluidic lab-on-achip betweet the top and the bottom plate. The top view depicts the position of the liquid access holes, the pogopins position and the screw holes.

syringe pump (Braun Perfusor Space) and by a peristaltic pump (Rainin RP-1 double channel) so to get three controlled liquid sppplies. 
The holder has six access holes $(1.25 \mathrm{~mm}$ of diameter) obtained by drilling the PMMA for liquid supply, sealed by PDMS rings. The connection with the pump tubing is made by commercial connectors, tightly fitting into the access holes. The electrical signals are provided by interfacing the substrate of the microfluidic chip to a small PCB placed on the top side of the holder. The electric contact is ensured by placing gold pogopins in contact with the PCB pads and the substrate ones. The pogopins are $1.15 \mathrm{~cm}$ long and the maximum travel length is $0.15 \mathrm{~cm}$ therefore the circuit holder thickness is set to $1 \mathrm{~cm}$. The microfluidic chip is tightly clamped between the upper part of the holder and a bottom cover with an M5 Teflon screw set.

\section{MICROFLUIDIC CHIP DESIGN AND FUNCTIONALITIES}

The microfluidic lab-on-a-chip is designed to be easily and rapidly configurable according to the needs. The design proposed includes a set of operations: mixing, focusing and trapping. Each of these operations is carried out thanks to a specific circuit layout. The combination of these functionalities is decided at the moment of engraving microfluidic channel layout and electrodes.

The microfluidic channels are obtained by soft lithography of PDMS. The standard height of the channels is between $8 \mu \mathrm{m}$ and $15 \mu \mathrm{m}$, special height of 100 to $300 \mu \mathrm{m}$ can be achieved by SU-8 standard photolithographic technology. The complete fabrication routine for the microchannels fabrication is shown in figure 2.

The microfluidic channels molding is obtained by spinning a thick layer of positive photoresist SPR220-7.0 (8 to $15 \mu \mathrm{m}$ depending on the recipe) on a 4 " borosilicate substrate. The photoresist is then ablated by laser direct writing using a 1064 nm YAG laser, which does not affect the glass substrate. The minimum channel width achieved is around 20 microns. The direct laser writing technique allows a fast prototyping and cost reduction of the microfluidic chips. UV lithography also allows good results but the minimum channel width is then 40 microns due to the resolution of the photolitography mask printer, and the costs rise up. It is furthermore possible to fabricate 100 to $300 \mu \mathrm{m}$ height microchannel using SU-8 2050 photoresist and the $1: 10$ aspect ratio must be respected leading to a wider channels result. Once the microchannels' mold is ready, the second step is pouring PDMS.

Usind the Sylgard 184 kit by Dow Corning, the polymer is mixed with the curing agent in a proportion of 10:1. Once poured, the PDMS is cured during 30 minutes at $70^{\circ} \mathrm{C}$. After the curing step, the PDMS is gently peeled off the glass substrate, washed with acetone and rinsed with water.

The access holes (fluidic and electric) are made using a 2 mm biopsy punch; the excess of PDMS is cut off using a sharp knife. The second part of the microfluidic chip, where electrodes lay, is made in PEN, which is a flexible, transparent and cheap plastic substrate, resistant to acids and biocompatible. The PEN is very suitable for metal deposition thanks to the high adhesion it shows. The PEN is spin coated with a photoresist. The patterns of the electrodes are directly engraved on the photoresist using the YAG laser to reduce the cost of photolithography masks. A thin metal film (generally gold) is deposited using an UNIVEX Magnetron Sputtering. After the deposition, the excess of metal is lifted off by photoresist wet etching.

Once the upper and lower sides of the microfluidic circuit are ready, they are irreversibly bonded by plasma oxidation of the PDMS and thermal treatment. Furthermore are described and analyzed some basic operations lab-on-a-chip can perform. The layouts are depicted and simulated. They can be combined into the microfluidic designing process according to the final needs.

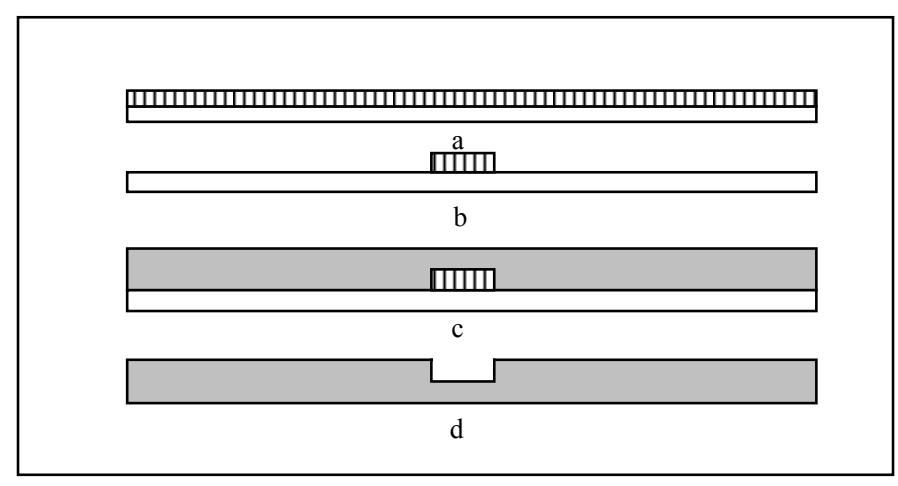

Figure 2. Microfluidic channels fabrication a) the photoresist is spinned on the substrate. b) The photoresist is ablated by laser direct writing (or processed by UV standard lithography). c) PDMS is poured on the mold and cured on a hot plate. d) the PDMS is gently peeled and the excess of silicon is cut off with a sharp knife.

The basic operations the chip is capable to run are: mixing and focusing the fluid flow and trapping and releasing micro and nano beads by electrostatic traps. For each operation is proposed a layout and the performances are evaluated by multiphysic simulations. The multiphysics simulations have been run using Comsol 3.5a, coupling the equations of the following modules:

\section{-Incompressible Navier-Stokes (mmglf) \\ -Conductive media DC (emdc) \\ -Convection and diffusion (ched).}

\section{A. Electrostatic mixing}

Mixing fluids at micro scale results to be very difficult because of the absence of turbulences due to the low Reynolds number and the laminar flow make the interfacial area of contact between molecules difficult to increase. Although it is sometimes necessary to efficiently and rapidly mix up two or more reagents [7] within a micro lab-on-a-chip, therefore mixing at micro scale becomes an important and difficult task. Several solutions have been presented during the paste decades [8-11]. The mixer implemented within the microfluidic lab-labon-a-chip is a based on electrostatic interactions with the laminar flow [8].

The layout of the mixer is based on a Y-shaped microfluidic circuit. Four electrodes are placed in the vicinity of two inlet channels exit and they are biased with an AC voltage of few volts and few hertz, figure 3 . 
In the model the two inlets are not represented. It is assumed at the entrance of the main channel, the flow is completely laminar, therefore the inlet boundary condition is given as the half-upper part of the inlet carries a $\mathrm{C} 0=1 \mathrm{~mol} / \mathrm{m}^{3}$ of reagent and the half-lower part of the stream carries a $\mathrm{C} 1=0$ $\mathrm{mol} / \mathrm{m}^{3}$. At the four electrodes is applied an AC voltage. These conditions make the surface of the microchannels to charge. The accumulation of charge at solid-liquid interface is

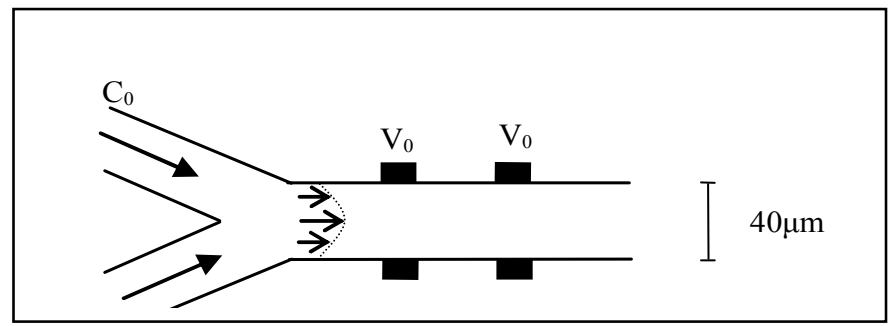

Figure 3. Electrooscmotic mixer layout: the flows go from left to right coflowing in the same mainstream channel. In the channel act four electrodes, biased with an AC voltage. The fluid stream is folded and stretched several times in order to create turbulences and swirls. The two fluids mix up efficiently and rapidly. No moving parts are involved in the process.

commonly known as electric double layer, which generates an electroosmotic flow, in this case locally turbulent.

The electroosmotic flow exerts a force on the charged solution close to the walls. The result is a flow in the direction of the electric field. The Helmotz-Smoluchowski equation[12] (1) describes well the velocity of the liquid flow in function of the tangential component of the electric field:

$$
u=\frac{\varepsilon_{W} \zeta_{0}}{\eta} \nabla_{T} V
$$

In the equation (1) $\xi_{W}$ is the fluid (water) electric permittivity $(\mathrm{F} / \mathrm{m}), \zeta_{0}$ is the zeta potential of the walls of the channel, and for PDMS it is $-23.1 \mathrm{mV}$ [13], $\eta$ is the dynamic viscosity of the fluid; at the walls is also applied the boundary condition of electroosmotic flow, taking in account the electric field at the boundary and the frequency of the voltage applied. These equations are valid on all the microchannels' walls, except the inlets and outlets.

The results applying an electric potential of $2.5 \mathrm{~V}$ at $10 \mathrm{~Hz}$ is shown in figure 4 . The fluid flows from left to right. The figure $4 \mathrm{a}$ and $4 \mathrm{~b}$ show flow trajectory at $\mathrm{t}=0.025 \mathrm{~s}, \mathrm{t}=0.075 \mathrm{~s}$. The trajectory of the fluid flow denotes an important swirling in the middle of the channel due to the electrodes' action. Due to the frequency, the rotation of the vortices changes with the time.

The fluid stream is stretched and folded several times due to the oscillation of the voltage applied. The laminar flow is locally perturbed resulting on an efficient and fast mixing of the fluid coming from the upper channel with the fluid coming from the lower one. The design of the microchannel is not affected. Only the electrodes design is taken in account to implement the mixing functionality. Figure $4 \mathrm{c}$ depicts the
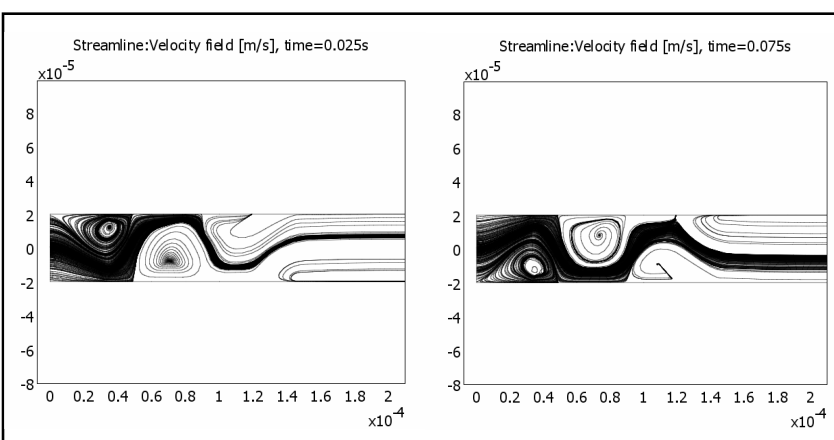

A

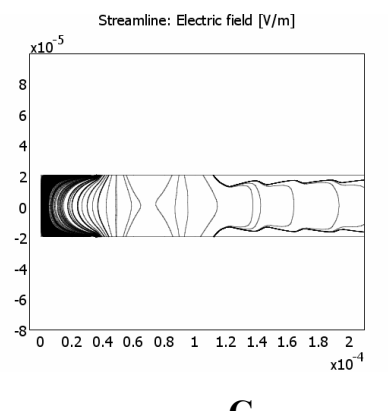

B

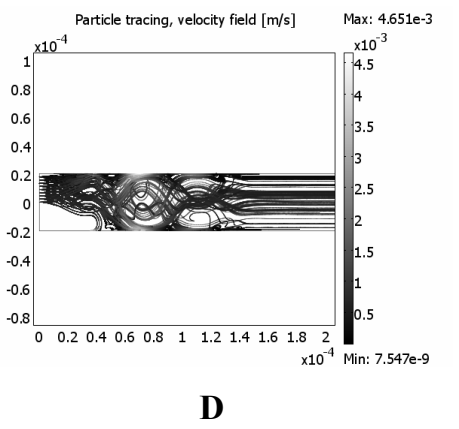

Figure 4. The fluid flows from left to right in the mainstream microchannel. a) and b) depict the fluid flow streamlines at $\mathrm{t}=0.025 \mathrm{~s}$ and $\mathrm{t}=0.075$. The fluid bends, stretches and folds several times due to the oscillations of the voltage applied. C) depicts the electric field in the microchannel while in d) are depicted the trajectories of particles flowing from the upper inlet channel; after passing the mixing area it is clearly homogeneous mixed with the flow coming from the lower channel.

electric field inside the microchannel while figure $4 \mathrm{~d}$ depicts a particle tracing plot in order to evaluate the effectiveness of the mixing process. From the simulation, a complete mixing is achieved in the range of $100 \mathrm{~ms}$.

\section{B. Hydrodynamic focusing}

In a microchannel the laminar condition of the flow is sometimes a limitation. Hydrodynamic focusing takes profit of this behavior by confining the main flow in the center of the channel introducing two lateral flows (and often an upper and lower flow) [14] in order to get the desired width of the central flow. A focused is central for many applications within the flow and particles' manipulation in a microfluidic lab-on-achip, such, cell counting, and sorting [15], trapping and ordering [16].

The layout for hydrodynamic focusing presented in this work consists in two $30^{\circ}$ tilted side flows coflowing in the main microchannel stream, with controlled fluid velocity by digital syringe pump.

The equation describing the flow behavior is: 


$$
\frac{w}{w_{0}}=\frac{v_{i}}{v_{i}+v_{f}}
$$

Where $w$ is the desired flow width, $w_{0}$ is the channel width; $v_{i}$ and $v_{f}$ are respectively the fluid velocity of the main inlet and the total lateral fluid flow of the focusing inlets. Equation (3) clearly shows the independence of the fluid focusing from the magnitude of the inlet. One can deal with fluid mass flow, pressure or velocity and the prediction is not affected.

The typical fluid velocity value applied is around $0.5 \mathrm{~mm} / \mathrm{s}$. In order to get a $2 \mu \mathrm{m}$ central fluid stream in a $40 \mu \mathrm{m}$ microchannel, the side focusing fluid velocity should be adjusted around $20 \mathrm{~mm} / \mathrm{s}$.

In figure 5 are shown some simulation results. The fluid velocity of the side channels is from 0.5 to 5 times higher than the mainstream fluid velocity $(0.5 \mathrm{~mm} / \mathrm{s})$. The microchannel width is $40 \mu \mathrm{m}$; the focused flow obtained goes from $20 \mu \mathrm{m}$ to $3.65 \mu \mathrm{m}$, figure $5 \mathrm{a}, 5 \mathrm{~b}$ and $5 \mathrm{c}$. Figure $5 \mathrm{~d}$ is a parametric plot of the focused centered flow in the channel $50 \mu \mathrm{m}$ away from the focusing area.

\section{Electrostatic traps of micro and nano beads.}

Particles and cells suspended in the fluid flow can be manipulated by applying a potential to specific sets of electrodes. Particles in colloidal form are usually charged. The surface charge makes the particle to interact with the electric field applied. Two main forces can take place in the trapping and releasing process: electrophoretic force [17] and dielectrophoretic force [18].

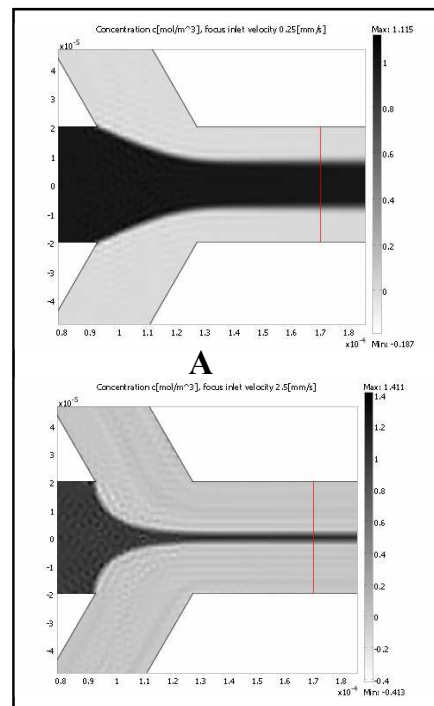

C

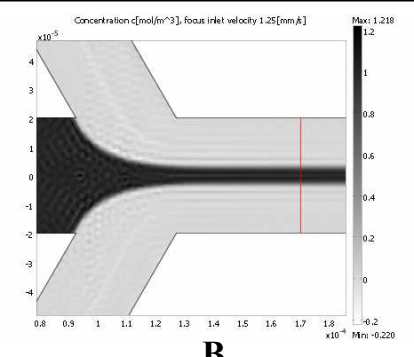

B

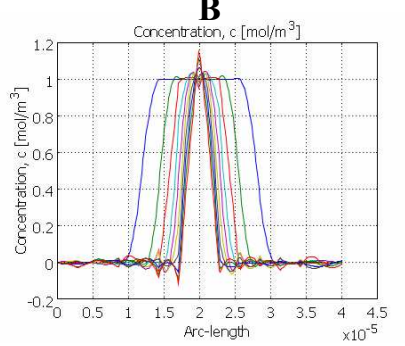

D
Figure 6. Fluid focusing: the mainstream is carried by the central microchannel at $0.5 \mathrm{~mm} / \mathrm{s}$. Two lateral flows act squeezing the central fluid flow and focusing is. A) depicts a focused flow of $20 \mu \mathrm{m}$ with a $0.25 \mathrm{~mm} / \mathrm{s}$ fluid velocity applied to each lateral inlet. B) The lateral flow is increased to $1.25 \mathrm{~mm} / \mathrm{s}$ resulting in a focused flow of $6.7 \mu \mathrm{m}$. C) shows a $3.65 \mu \mathrm{m}$ focused flow. D) is a parametric plot of the focused centered flow $50 \mu \mathrm{m}$ away from the focusing area

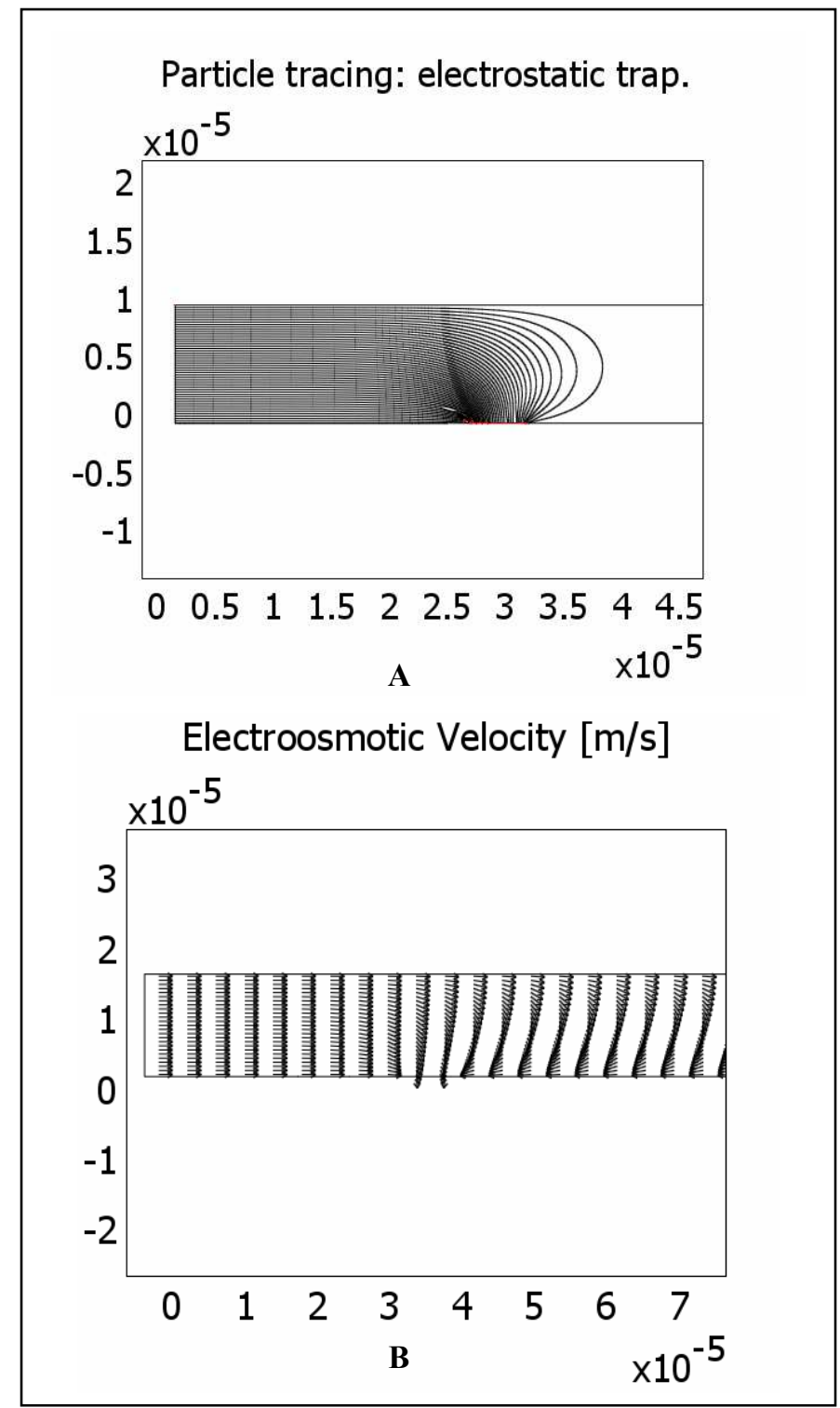

Figure 5. Trapping of particles in the fluid stream (from left to right) by electrostatic means: a) depicts the particle tracing plot. Particles are considered negatively charged. They are trapped by a positive biased set of electrodes. B) shows the electroosmotic velocity plot inside the microfluidic channel.

The solution proposed in this work is based on interdigitated electrodes. It is assumed the gravity is negligeable as the particle density is close to the fluid's one $\left(1.05 \mathrm{~g} / \mathrm{m}^{3}\right)$. The electrophoretic trapping consists on applying DC voltage to one electrode. The particles dispersed in the fluid have a surface charge. According to the Smoluchowski double layer theory [19], the electrostatic Coulomb force exerted by the electric field on the particles is:

$$
\vec{F}_{E P}=6 \pi \varepsilon_{w} r^{3} \xi_{P} \vec{E}
$$

In equation (3) $r$ is the radius of the particle $\varepsilon_{\mathrm{w}}$ is the permittivity of the liquid buffer, $\xi_{\mathrm{P}}$ is the zeta potential of the particle and $\vec{E}$ the induced electric field. 
The electroosmotic velocity of the particle, due to the electric field is:

$$
U_{E O F}=-\frac{\varepsilon_{p} \xi_{p}}{\eta} \vec{E}
$$

In equation (4) $\eta$ is the dynamic viscosity of the liquid. In figure $6 \mathrm{a}$ are depicted the particle tracing streamlines. The particles are trapped by the bottom electrode when activated. In figure $6 \mathrm{~b}$ is plot the electroosmotic velocity of the flow. The arrows clearly depict the particles are dragged towards the bottom area where the electrode is placed.

The dielectrophoresys is a phenomenon involving micro and nano particles in a fluid interacting with a non-uniform electric field that induces on the particles a polarization charge [20-22].

The intensity and the force field depend on the voltage applied, frequency, dielectric properties of the particles and solution and electrodes configuration.

The movement of the particles towards the strong electric field is generally called positive dielectrohoresys (pDEP)[23]; the movement towards the opposite direction is called negative dielectrophoresys (nDEP)[24].

Applying an AC voltage to a set of interdigitated electrodes, the force exerted on a dielectric particle is:

$$
F_{D E P}=2 \pi \varepsilon_{m} r^{3} \operatorname{Re}(K(\omega)) \nabla E_{r m s}^{2}
$$

In equation (5) $\varepsilon_{m}$ is the dielectric permittivity of the suspended medium $(\mathrm{F} / \mathrm{m}), r^{3}$ is the particles radius $(\mathrm{m}), E^{2}$ is the root-mean-square $(\mathrm{rms})$ of the electric field $(\mathrm{V} / \mathrm{m})$ and $\operatorname{Re}(K(\omega))$ is the real part of the Clausius-Mossotti factor.

The Clausius-Mossotti factor describes

$$
\begin{array}{r}
\text { CM factor } \quad 0.35 \text { at } 1 \text { megaherz } \\
\text { Ndep } 0.98
\end{array}
$$

\section{CONCLUSIONS}

The work presented in this paper is aimed to report the design and the multiphysic simulations of a cost effective microfluidic platform with liquid and electric interfaces with a flexible, biocompatible and versatile microfluidic lab-on-achip. The interface platform allows fast and versatile connections. The electric signals can be collected and applied by a simple PCB interface and pogopins connections.

The microfluidic lab-on-a-chip can perform the most important functions for the manipulation of particles in fluid streams. Each function has a standard layout. At the moment of the device fabrication by direct laser writing, the overall layout can be decided on demand.

The results obtained from simulations show a rapid and efficient electroosmotic mixing in the hundred of milliseconds range. It is possible to easily focusing a flow by adjusting the lateral flow inside the channel. Therefore the particle focusing, sorting and counting can be easily performed thanks to the electrode sets available. Electrodes also provide a powerful trapping device, based on electrophoretic or dielectrophoretic trapping and release. The particles and cells can be trapped and optical measurement can be run on the trapping sites.

\section{ACKNOWLEDGMENT}

This work has been founded by the Spanish Ministry of Science and Innovation with the grant BES-2008-007481 associated to the project TEC2007-67081.

\section{REFERENCES}

[1] Brian M Paegel, Robert G Blazej, Richard A Mathies "Microfluidic devices for DNA sequencing: sample preparation and electrophoretic analysis Review Article", Current Opinion in Biotechnology, Vol.14, 1, 2003, P.42-50.

[2] Eric T. Lagally, Peter C. Simpson, Richard A. Mathies, "Monolithic integrated microfluidic DNA amplification and capillary electrophoresis analysis system", Sensors and Actuators B: Chemical, Vol. 63,3, 2000, P.138-146.

[3] S.L. Lonigro, F. Valerio, M. De Angelis, P. De Bellis, P. Lavermicocca, "Microfluidic technology applied to cell-wall protein analysis of olive related lactic acid bacteria“, International Journal of Food Microbiology, Vol.130,1, 2009, P.6-11

[4] Y.Song, H. Zhang, C.H.Chon, S.Chen, X.Pan, D. Li, "Counting Bacteria on a Microfluidic Chip", Anal Chim Acta. 2010 Nov 29;681(1-2):82-6. Epub 2010 Sep 29

[5] P.Chen, X.Feng, R.Hu, J.Sun, W.Du, B.F.Liu, "Hydrodynamic gating valve for microfluidic fluorescence-activated cell sorting", Anal. Chim. Acta, Vol.6,63,1, 2010, P.1-6.

[6] J.Mairhofer, K. Roppert and P.Ertl, "Microfluidic Systems for Pathogen Sensing: A Review", Sensors 2009, 9(6), P.4804-4823.

[7] R. Liu, R. Lenigk, R.L Druyor-Sanchez, J. Yang, P. Grodzinski, "Hybridization enhancement using cavitation microstreaming", Analyt. Chem. 2003, 75, 1911-1917.

[8] M.Oddy, J. Santiago, J. Mikkelsen, "Electrokinetic instability micromixing“, Analyt. Chem. 2001, 73, 5822-5832.

[9] J. Knight, A. Vishwanath, J.P. Brody, R.H. Austin,. "Hydrodynamic focusing on a silicon chip: mixing nanoliters in microseconds", Phys. Rev. Lett. 1998, 80, 3863-3866.

[10] W. Ehrfeld, J. Hartmann, V. Hessel, S. Kiesewalter, H. Lowe, "Microreaction technology for process intensification and high throughput screening”, In Proc. Micro Total Analysis Systems Symp. 2000a p. 33-40.

[11] W. Ehrfeld, H. Lowe, V. Hessel, "Microreactors: new technology for modern chemistry", 1st edn., 2000b Wiley-VCH.

[12] R.J. Hunter, Zeta Potential in Colloid Science, Academic Press, New York, 1981.

[13] Ren X., Bachman M. et al., "Electroosmotic properties of microfluidic channels composed of poly(dimethylsiloxane)", Journal of Chromatography B: Biomedical Sciences and Applications (2001), A 762, 117.

[14] T. Stiles, R. Fallon, T. Vestad, J. Oakey, D. W. M. Marr, J. Squier, and R. Jimenez, "Hydrodynamic focusing for vacuum-pumped microfluidics," Microfluidics and Nanofluidics 2005, No.1, pp.280-283.

[15] Yang S-Y, Hsiung S-K, Hung Y-C, Chang C-M, Liao T-L, Lee G-B, “A cell counting/sorting system incorporated with a microfabricated flow cytometer chip", Meas. Sci. Technol. (2006), 17(7):2001-2009

[16] Lutz, B.R.; Chen, J.; \& Schwartz, D.T. "Hydrodynamic tweezers: noncontact trapping of single cells using steady streaming microeddies", Analytical Chemistry, 78, 5429-5435, 2006.

[17] Yasukawa T, Nagamine K, Horiguchi Y, Shiku H, Koide M, Itayama T, Shiraishi F, Matsue T. 2008. "Electrophoretic cell manipulation and electrochemical gene-function analysis based on a yeast two-hybrid system in a microfluidic device", Anal Chem 80(10):3722-3727. 
[18] Sachiko Ogata, Tomoyuki Yasukawa, Tomokazu Matsue, "Dielectrophoretic manipulation of a single chlorella cell with dualmicrodisk electrode", Bioelectrochemistry, Volume 54, Issue 1, August 2001, Pages 33-37,

[19] J. Lyklema, "Electrokinetic after Smoluchowski", Colloids and Surfaces A: Physicochemical and Engineering Aspects, 2003, V. 222, Issues 1-3, Pages 5-14

[20] Pohl, H. A. Dielectrophoresis; Cambridge University Press: Cambridge, U.K., 1978.
[21] Jones, T. B. Electromechanics of particles; Cambridge University Press: New York, 1995.

[22] Morgan, H.; Green, N. G. AC Electrokinetics: Colloids and nanoparticles;AResearchStudiesPressBook: Baldock,Hertfordshire, England, 2003.

[23] Hughes, M. P. Electrophoresis 2002, 23, 2569-2582.

[24] Matsumoto, N.; Matsue, T.; Uchida, I. Bioelectrochem. Bioenerg. 1994, 34, 199-202. 